\title{
A Web-based Integrated Medical Information System for Cholangiocarcinoma Screening and Periductal Fibrosis Ultrasound Image Processing
}

\author{
Piyamas Suapang ${ }^{\mathrm{a},{ }^{*},}$, Wisarut Krissana ${ }^{\mathrm{a}}$, Prasong Tosranon ${ }^{\mathrm{b}}$ \\ a'Biomedical Engineering Faculty, Rangsit University, Pathumthani, 12000 Thailand \\ ${ }^{\mathrm{b}}$ Department of Industrial Physics and Medical Instrumentation, King Mongkut's University of Technology North \\ Bangkok, Bangkok, 10800, Thailand \\ *Corresponding Author: piyamas_suapang@.yahoo.com
}

\begin{abstract}
Cholangiocarcinoma is the most common in northeast Thailand and the second of most common primary liver cancer worldwide. As an online information system, it reduces the burden more clinical research and education are required and eliminates the restrictions of the traditional isolated PACS workstations. In addition, The periductal fibrosis ultrasound images can be applied for the cholangiocarcinoma screening system. However, the ultrasound images are lack of contrast and a lot of speckle noise. This study developed a web-based integrated clinical information system and periductal fibrosis ultrasound image processing for cholangiocarcinoma to enable rapid access to both digital images and associated text reports for audit, education and research. Emphasizing confidentiality of anonymised data and access control, the system provides a virtual collaboration platform integrating various clinical data sets for research and continuing education. What's more, the study presented image processing algorithm to improve the lack of contrast, reduce the speckle noise and preserving the edge, smooth region in periductal fibrosis ultrasound image. The system can potentially support remote consulting and statistical analysis of aggregated multimodality data. Furthermore, the performance of algorithm from the experimental results shown that increase the performance for enhancing the periductal fibrosis ultrasound image for cholangiocarcinoma screening system.
\end{abstract}

Keywords: Medical Information System, Ultrasound Image Processing, Cholangiocarcinoma.

\section{Introduction}

The humans are ingesting the freshwater fish which has the parasite, or liver fluke. The liver fluke may cause chronic inflammation of the bile ducts and periductal fibrosis which may subsequently lead to the development of cholangiocarcinoma. Cholangiocarcinoma is the most common in northeast Thailand that is second of most common primary liver cancer worldwide. The parasitic infestation, primary sclerosing cholangitis, biliary duct cyst, hepatolithesis and toxins are factors of cholangiocarcinoma. In Thailand, the parasitic infestation is an important risk factor for cholangiocarcinoma which corresponds to the endemic area of the liver fluke, Opisthorchis viverrini. Ultrasound basically has major role in detection, diagnosis of liver and biliary disease and assessment of liver parenchyma. Ultrasound is less invasiveness, less harm, easy availability and easy portability.

In today's health care system, there are many uses of teleradiology. One is to, "provide urgent services at sites either without a radiologist or physician requiring back- up". Secondly, teleradiology can, "Provide immediate access to subspecialty radiology consultants". Thirdly, and probably the one most often used, is to, "generate second opinions". And finally, teleradiology can be used to, continue medical education, proficiency, testing, and recertification of radiologists as well as other laboratory personnel". This can be extremely beneficial to students in rural areas. This way, they do not have to travel far to receive their education or take exams. Obviously, teleradiology has become an 
important aspect of telemedicine in the past few years and it will even more in the years to come. With the advent of computer and communication technologies, construction of an effective teleradiology system becomes possible [1].

Picture archiving and communication system (PACS) is widely used to manage DICOM files. PACS provides the mechanism for data communication, transmission, storage, and connection to existing hospital information systems (HIS) or radiology information systems (RIS). However, system integration generally lags behind state of the art imaging and storage facilities. In part, this may arise because industrial objectives are often dictated by commercial aims rather than attempts at optimizing the end user experience [2]. In particular, information retrieval from disparate and large data sets in hospitals remains problematic. Often a query of RIS or PACS database is limited by a search for individual patients either by name or identification number [3, 4]. At best, a query returns imaging performed on a particular date or under a general category which includes attending physician and location. Furthermore, DICOM image viewers often do not take full advantage of web-related innovations.

The periductal fibrosis ultrasound images can be applied for the cholangiocarcinoma screening system which increased patient survivability [5-8]. However, the ultrasound images are lack of contrast and a lot of speckle noise. Joo et al. [9] proposed a computer-aided diagnosis (CAD) of solid breast nodules. The enhancement methods are combination of the median filter, unsharp masking, and the contrast stretching transformation. Shi et al. [10] presented the detection and classification of masses in breast ultrasound images method. In this research used generalized histogram equalization, Markov random field, and Gibbs random field for enhancing the ultrasound images. Jumaat et al. [11] proposed the segmentation of masses from breast ultrasound images using parametric active contour algorithm. In this research used the median filter and histogram stretching method for enhancing the ultrasound images. Su et al. [12] presented the automatic detection and classification of breast tumors in ultrasound images using texture and morphological features. In this research used the speckling reducing anisotropic diffusion algorithm, gradient operator and Laplace operator for enhancing the ultrasound images.

In this report describe design and implementation of a web-based integrated clinical information system for cholangiocarcinoma to collect clinically relevant data in patient care, such as physiological parameters, laboratory results, and radiological imaging are often maintained separately as physicians have to integrate all clinical data in daily decision making. Furthermore, this study proposed periductal fibrosis ultrasound image processing for cholangiocarcinoma to improve the lack of contrast, reduce the speckle noise and preserving the edge, smooth region in periductal fibrosis ultrasound image.

\section{Methodology}

\subsection{Overview of Web-based Integrated Clinical Information System for Cholangiocarcinoma}

Bearing in mind that the system under consideration is a web-based integrated clinical information system for cholangiocarcinoma, the choices in terms of development platform should comprehend distributed components under a client-server architecture. One of the most important requirements for the system is that the application be completely web-based. This way, it becomes much more portable and sustainable. On top of that, web-based technologies allow the system to become available virtually from everywhere at anytime, a critical factor for medical applications where a few hours could represent the difference between a late diagnosis and saving a life. A Radiology Information System (RIS) is in charge of updating the patients information along with the image associated to them. This is carried out using a database (MySQL), that a reliable and proven relational database, was used to manage both text and image data. Apache 2.0 HTTP server and PHP scripting engine were implemented to provide web-based user interface function. The system architecture is illustrated in Figure 1. Digital versions of patient clinical summaries were assembled from the gastroenterology department databank. These data are collectively termed input data. Advantage of MySQL's search capability, all information extracted from text reports and annotations are searchable in a web-based integrated clinical information system for cholangiocarcinoma. A web-based integrated clinical information system for cholangiocarcinoma provides multiple options for searches. Search by category is designed for diagnosis search. Search by reports offers key word search in various subjects. Search by study enables group collection according to examination date or ultrasound serial number. Search by note is suited for annotation query. Furthermore, we implemented both Boolean operators and wild-card in search engine to enable flexible and comprehensive 
retrieval. Text files were entered into the system, then parsed and classified. Classification was based on enrollment, demographic information, ultrasound screening, diagnosis and treatment, follow-up treatment, final staging diagnosis and post operation follow-up. The text portion of a web-based integrated clinical information system for cholangiocarcinoma was categorized into various fields which were linked through patient ID. All patient data that might allow identification of individual persons were encrypted to protect patient confidentiality and privacy. This was done by using symmetric key algorithms to generate ciphers for all real identifiers. The resulting anonymous identifiers were used to replace the actual identifiers before they were stored in a web-based integrated clinical information system for cholangiocarcinoma.

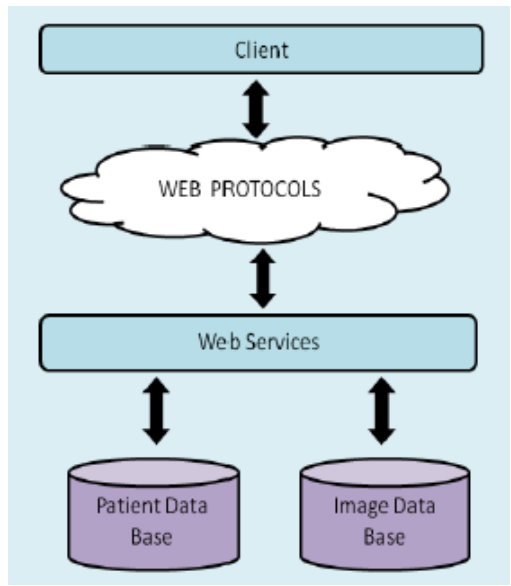

Fig. 1. Main components of a web-based integrated clinical information system for cholangiocarcinoma.

\subsection{Periductal Fibrosis Ultrasound Image Processing for Cholangiocarcinoma}

Periductal fibrosis ultrasound image processing consisted of the contrast improvement, reduce the speckle noise and preserving the edge, smooth region in periductal fibrosis ultrasound image. The periductal fibrosis ultrasound images are lack of contrast. In this paper applied Local Contrast Enhancement (as in equation (1)) to improve the periductal fibrosis ultrasound images which can improve lack of contrast of the periductal fibrosis ultrasound images. With local contrast enhancement, intensity is adjusted more clearly while the colour remains original. A clearer image will be produced.

$$
f(i, j) \rightarrow g(i, j)=255 \frac{\left|\psi_{w}(f)-\psi_{w}\left(f_{\min }\right)\right|}{\left|\psi_{w}\left(f_{\max }\right)-\psi_{w}\left(f_{\min }\right)\right|}
$$

where the sigmoid function is $\psi_{w}(f)=\left[1+\exp \left(\frac{\langle f\rangle_{w}-f}{\sigma_{w}}\right)\right]^{-1}$ while $f_{\max }$ and $\min f_{\min }$ are the maximum and minimum value of intensity within the whole image with $\langle f\rangle_{w(i, j)}=\frac{1}{M^{2}} \sum_{(k, l) \in w(i, j)} f(k, l) \quad$ and $\sigma_{w}^{2}(f)=\frac{1}{M^{2}} \sum_{(k, l) \in(i, j)}\left(f(k, l)-\langle f\rangle_{w}\right)^{2}$

The periductal fibrosis ultrasound images have issue of speckle noise in which added difficulty to detect the periductal fibrosis region for the cholangiocarcinoma screening system. Furthermore, the speckle noise can decrease the periductal fibrosis detection system in which can identify the group of the cholangiocarcinoma. In this paper applied the speckle reduction method in model which consisted of the fast fourier transform and median filter methods. The removal of impulse noise involves median filter, which is conceptually similar to that of the mean/average filter. The processing pixel is replaced by median of the $3 \times 3$ neighbourhood. If the selected $3 \times 3$ window is $\mathrm{W}=\{12,123,0,56, \boldsymbol{0}, 78,45,55,55\}$ with the processing pixel as ' 0 ', then the window elements are arranged in ascending order $(\mathrm{W}=\{0,0,12,45, \mathbf{5 5}, 55,56$, $78,123\}$ ), and the mid value is taken as median (i.e 55), and the processing pixel ' 0 ' will be replaced by the median value ' 55 '. The major advantage of median filter is that it can preserve details of the image such as edges and contours. In this step can reduce the speckled and preserved the edge of the periductal fibrosis ultrasound images.

The periductal fibrosis ultrasound images are lack of contrast in intraregion in which can decrease the performance of the periductal fibrosis detection. In this paper applied the anisotropic diffusion [17] to smooth region of the periductal fibrosis ultrasound images. Anisotropic diffusion proposed the following nonlinear periductal fibrosis for smoothing image on a continuous domain:

$$
\left\{\begin{array}{l}
\frac{\partial I}{\partial t}=\operatorname{div}[c(|\nabla I|) \cdot \nabla I] \\
I(t=0)=I_{o}
\end{array}\right.
$$

where $\nabla$ is the gradient operator, the divergence operator, 
denotes the magnitude, $c(x)$ the diffusion coefficient,

and $I_{o}$ the initial image. They suggested two diffusion coefficients

$$
c(x)=\frac{1}{1+(x / k)^{2}}
$$

and

$$
c(x)=\exp \left[-(x / k)^{2}\right]
$$

where $k$ is an edge magnitude parameter. In the anisotropic diffusion method, the gradient magnitude is used to detect an image edge or boundary as a step discontinuity in intensity. If $|\nabla I|\rangle\rangle k$, then $c(|\nabla I|) \rightarrow 0$, and we have an all-pass filter; if $|\nabla I|\langle\langle k$, then $c(|\nabla I|) \rightarrow 1$, and we achieve isotropic diffusion (Gaussian filtering). A discrete form of (1) is given by

$$
I_{s}^{t+\Delta t}=I_{s}^{t}+\frac{\Delta t}{\bar{\eta}_{s}} \sum_{p \in \bar{\eta}_{s}} c\left(\nabla I_{s, p}^{t}\right) \nabla I_{s, p}^{t}
$$

where is $I_{s}^{t}$ the discretely sampled image, $s$ denotes the pixel position in a discrete two-dimensional (2-D) grid, and $\Delta t$ is the time step size, $\bar{\eta}_{s}$ represents the spatial neighborhood of pixel $s,\left|\bar{\eta}_{s}\right|$ is the number of pixels in the window (usually four, except at the image boundaries), and $\nabla I_{s, p}^{t}=I_{p}^{t}-I_{s}^{t}, \quad \forall_{p} \in \bar{\eta}_{s}$.

The advantages of anisotropic diffusion include intra-region smoothing and edge preservation. Anisotropic diffusion performs well for images corrupted by additive noise. Several enhancements and edge detection methods have been described in the literature [18] for images with additive noise. In cases where images contain speckle, anisotropic diffusion will actually enhance the speckle, instead of eliminating the corruption. The work of this paper uses the strengths of the PDE approach to produce edge-sensitive speckle reduction.

\section{Results and Discussion}

Using a web server to archive the radiology data and medical records, a web-based integrated clinical information system for cholangiocarcinoma does not require any client side computer software installation or customisation. As an online databank it could be accessed through any operating system. It allows multiple user access in real time. This can be of particularly helpful after hours. It provides a platform by which residents can discuss a case with the attending neurosurgeons without being confined to the same geographic location, and may be useful for institutions which do not have PACS access from home. The aim of a web-based integrated clinical information system for cholangiocarcinoma is to create an online integrated information system for a cohort of patients with cholangiocarcinoma in order to support surveillance, clinical research and facilitate professional education. A web-based integrated clinical information system for cholangiocarcinoma is a content-rich online resource for cholangiocarcinoma research and education. It also offers a suite of interactive functions for clinicians and researchers to retrieve, visualize, capture, exchange and share both text and image data through simple intuitive interface. User-friendliness and intuitiveness are two key features that busy clinicians desire in any research and educational computer system. In order to promote interaction, facilitate comprehensive information retrieval and image annotation, a web-based integrated clinical information system for cholangiocarcinoma enables multiple search options, visualization choices, and online updates. Boolean operators are implemented to facilitate flexible and compound search. For example user is able to do a compound search, such as only male patient age between 31 to 65 years old. Functional features of a web-based integrated clinical information system for cholangiocarcinoma are summarized in Table 1. Figure 2 illustrates the features of "Full report" page. Digital images and text documents are presented side-by-side for ease of comparison. The primary users of a web-based integrated clinical information system for cholangiocarcinoma are physician, radiologists, and medical students. It is a proof of concept web-based integrated knowledge system for cholangiocarcinoma, which designed to facilitate clinical research and teaching. This research focused on 
cholangiocarcinoma as it is one of the leading causes of death and favourable outcome is achievable by systematic research and improving treatment strategy. A web-based integrated clinical information system for cholangiocarcinoma related research often requires maintenance of an extensive database because of the numerous variables encountered. Incorporating digital images into such database was difficult in the past, and a web-based integrated clinical information system for cholangiocarcinoma is designed to allow unlimited incorporation of both text and image datasets.

Table 1. The Functional features of a web-based integrated clinical information system for cholangiocarcinoma

\begin{tabular}{|c|c|}
\hline Web page & Contents \\
\hline Home & Brief introduction of the system; Online tutorial \\
\hline Browser & $\begin{array}{l}\text { Patient profile including diagnosis and severity. } \\
\text { Patients' identification is encrypted from } \\
\text { regular user viewing. Options to view text } \\
\text { report, image or full report. }\end{array}$ \\
\hline Search Engine & $\begin{array}{l}\text { Search options for different tables in a } \\
\text { web-based integrated clinical information } \\
\text { system for cholangiocarcinoma with clear } \\
\text { guidance and labels. } \\
\text { Search categories: diagnostic categories with } \\
\text { Boolean operators. In addition, patient's age can } \\
\text { be used to narrow the search range. } \\
\text { Search text reports: key word search with } \\
\text { "wild-card" character. } \\
\text { Search study: search according to US date, or a } \\
\text { specific case search using study ID. } \\
\text { Search note: options to search notes in a time } \\
\text { frame or based on user name, or subject of } \\
\text { content. }\end{array}$ \\
\hline Image Report & $\begin{array}{l}\text { Options to view images individually or in a } \\
\text { group; All instances can be annotated } \\
\text { individually or as a set; Annotation can be } \\
\text { updated, added or deleted online. }\end{array}$ \\
\hline Text Report & $\begin{array}{l}\text { Power user can edit and update "doctor's } \\
\text { comments", "diagnoses" and "Stage". }\end{array}$ \\
\hline Statistics & $\begin{array}{l}\text { Summary of diagnostic information in the } \\
\text { database. }\end{array}$ \\
\hline Document & Details of user instruction for the system. \\
\hline
\end{tabular}
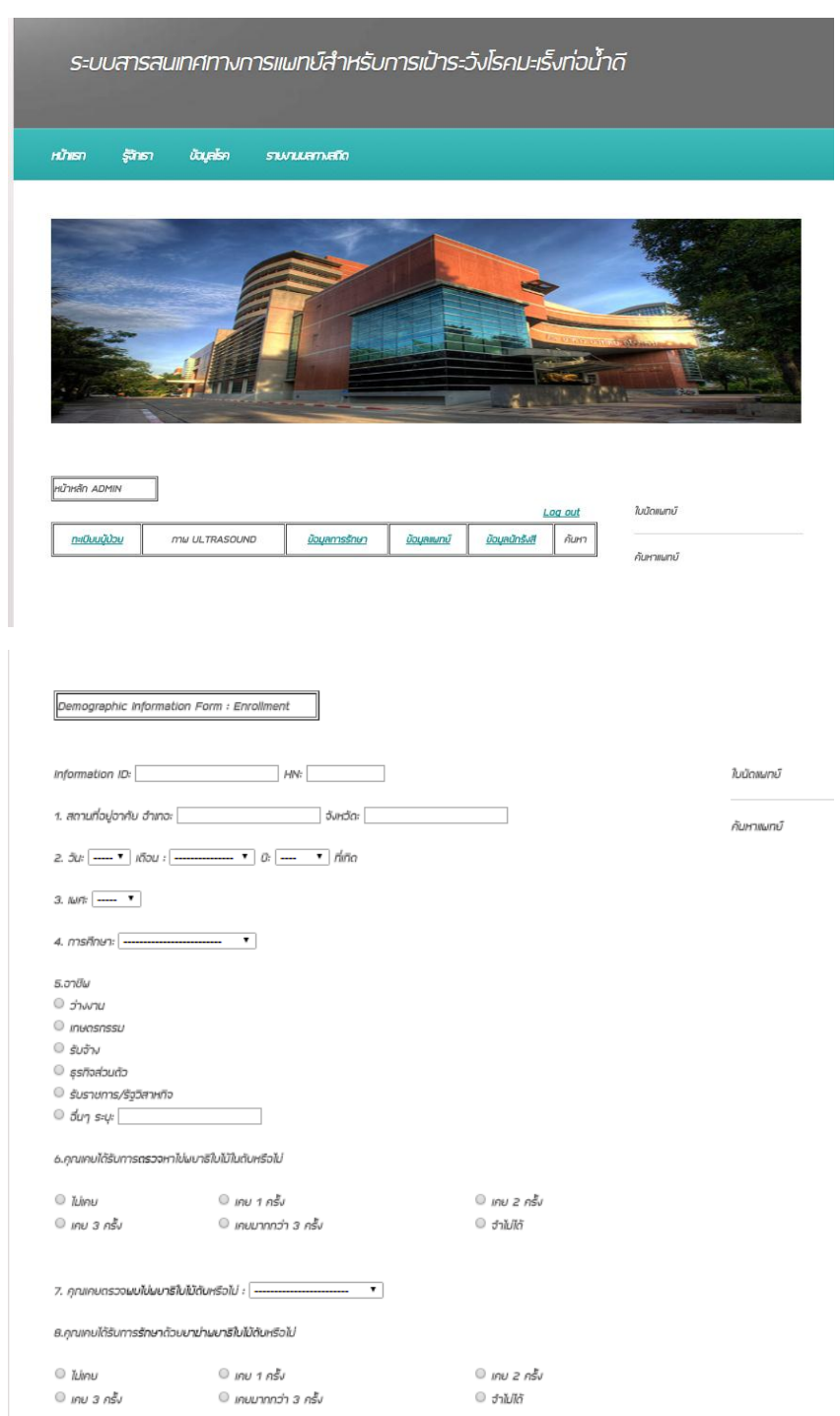

Fig. 2. A web-based integrated clinical information system for cholangiocarcinoma and report generator.

In this study used the periductal fibrosis ultrasound images 30 images with the size of the periductal fibrosis ultrasound images are $300 \times 300$ pixels for experimentation of periductal fibrosis ultrasound image processing for cholangiocarcinoma screening using the local contrast enhancement, speckle reduction method, and anisotropic diffusion. The sample results of the periductal fibrosis ultrasound images after local contrast enhancement, speckle reduction method and smoothing region are shown in fig. 3, 4 and 5 , respectively. 


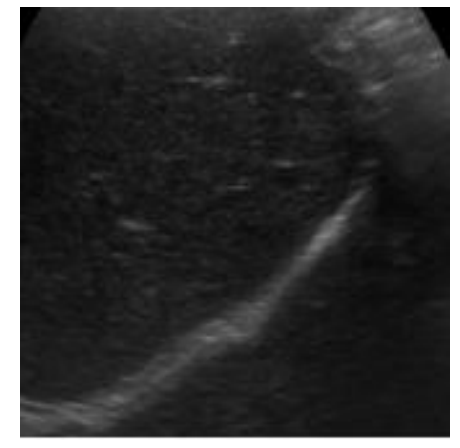

(a)

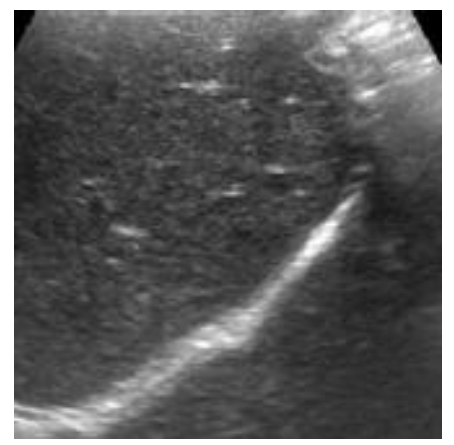

(b)

Fig. 3. Example result images of local contrast enhancement (a) original periductal fibrosis image (b) using the local contrast enhancement.

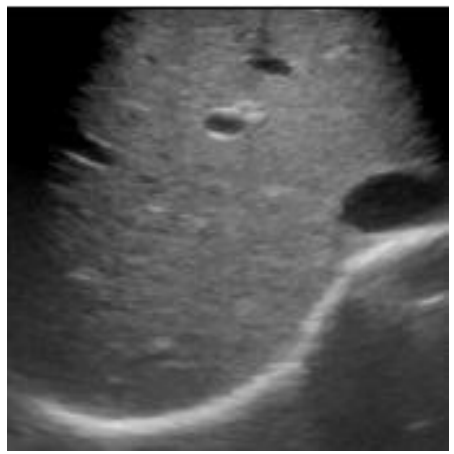

(a)

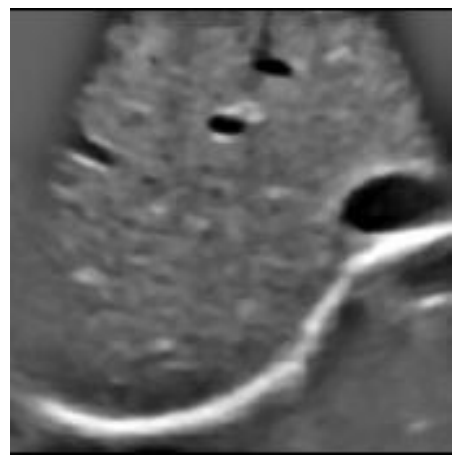

(b)

Fig. 4. Example result images of speckle reduction method (a) original periductal fibrosis image (b) using the speckle reduction method.

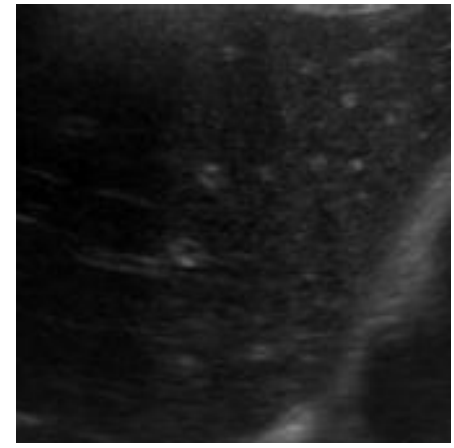

(a)

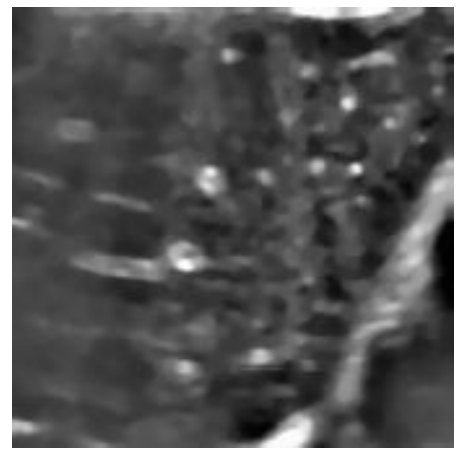

(b)

Fig. 5. Example result images of anisotropic diffusion method (a) original $\mathrm{v}$ image (b) using the anisotropic diffusion.

Figure 3-5. show the example of periductal fibrosis ultrasound images after enhancement with the methods. The method has high performance can improve lack of contrast of the periductal fibrosis ultrasound images. It can reduce the speckle noise and smoothed region in the periductal fibrosis ultrasound images. So, the periductal fibrosis ultrasound image result of the method has low speckle noise after segmentation (see Fig. 4 (b)). The method can increase the performance of the periductal fibrosis detection system because the method applied the local contrast enhancement method to improve the contrast, reduced speckle noise by model and smoothed periductal fibrosis region by anisotropic diffusion method.

\section{Conclusions}

This report describes the development of a web-based integrated clinical information system for cholangiocarcinoma, a centralized online medical information system for cholangiocarcinoma research and education. The significant features of a web-based integrated clinical information system for cholangiocarcinoma includes 1) Integration of US images and text reports into a single database; 2) Multiple search 
options enhancing retrieval capability; 3) A user-friendly interface promotes clinical research and online learning. A web-based integrated clinical information system for cholangiocarcinoma has been developed and showed the usefulness. Furthermore, in this paper proposed the technique for enhancement of periductal fibrosis ultrasound images for cholangiocarcinoma screening. In this technique consist of the local contrast enhancement, speckle reduction method, and anisotropic diffusion. The experimental results demonstrated the performance of the method in which improved the lack of contrast, reduced speckle noise, and preserved the edges periductal fibrosis images. The method can increase the performance of the periductal fibrosis detection system for cholangiocarcinoma screenig. In the future, we will plan to increase the performance of the method in the periductal fibrosis detection system and performance comparison of difference enhancement method.

\section{Acknowledgment}

This work is partially supported by Biomedical Engineering Faculty, Rangsit Research Institute at Rangsit University and Department of Industry Physics and Medical Instrument at King Mongkut's University of Technology North Bangkok. The authors also gratefully acknowledge the helpful comments and suggestions of the reviewers, which have improved the presentation.

\section{References}

(1) Richard Wootton, Nivritti G Patil, Richard E Scott, Kendall Ho, "Telehealth in the Developing World", Royal Society of Medicine Press Ltd., 2009.

(2) Bhattathiri PS, Gregson B, Prasad KS, Mitchell P, Soh C, Mitra D, et al: Reliability assessment of computerized tomography scanning measurements in intracerebral hematoma. Neurosurg Focus. 15:E6, 2003.

(3) Lim CC, Yang GL, Nowinski WL, Hui F: Medical Image Resource Center--making electronic teaching files from PACS. J Digit Imaging. 16:331-336, 2003.

(4) Minati L, Ghielmetti F, Ciobanu V, D'Incerti L, Maccagnano C, Bizzi A, Bruzzone MG: Bio-image warehouse system: concept and implementation of a diagnosis-based data warehouse for advanced imaging modalities in neuroradiology. J. Digit. Imaging. 20:32-41, 2007.

(5) N. Chamadol, C. Pairojkul, N. Khuntikeo, V. Laopaiboon, W. Loilome, P. Sithithaworn, and P. Yongvanit, "Histological confirmation of periductal fibrosis from ultrasound diagnosis in cholangiocarcinoma patients", Journal of Hepato-Biliary-Pancreatic Sciences, vol. 21, 316-322, Jan 132014.

(6) G. Tyson and H. El-Serag, "Risk factors for cholangiocarcinoma", Hepatology, vol. 54, 173-184, 2011.
(7) B. Sripa, E. Mairiang, B. Thinkhamrop, T. Laha, S. Kaewkes, P. Sithithaworn, S. Tessana, A. Loukas, P. Brindley, and J. Bethony, "Advanced periductal fibrosis from infection with the carcinogenic human liver fluke Opisthorchis viverrini correlates with elevated levels of interleukin-6", Hepatology, vol. 50, 1273-1281, 2009.

(8) S. A. Khan, B. R. Davidson, R. Goldin, S. P. Pereira, W. M. Rosenberg, S. D. Taylor-Robinson, A. V. Thillainayagam, H. C. Thomas, M. R. Thursz, and H. Wasan. (2002). British Society of Gastroenterology Guidelines for the diagnosis and treatment of cholangiocarcinoma. 61.

(9) S. Joo, Y. S. Yang, W. K. Moon, and H. C. Kim, "Computer-Aided Diagnosis of Solid Breast Nodules: Use of an Artificial Neural Network Based on Multiple Sonographic Features," IEEE Transactions on Medical Imaging, vol. 23, 1292-1300, 2004.

(10) X. Shi, H. D. Cheng, L. Hua, W. Jua, and J. Tian, "Detection and Classification of Masses in Breast Ultrasound Images," Digital Signal Processing, vol. 20, 824-836, 2010.

(11) A. K. Jumaat, W. E. Z. W. A. Rahman, A. Ibrahim, and R. Mahmud, "Segmentation of Masses from Breast Ultrasound Images using Parametric Active Contour Algorithm," in International Conference on Mathematics Education Research 2010 (ICMER 2010), 2010, 640-647.

(12) Y. Su, Y. Wang, J. Jiao, and Y. Guo, "Automatic Detection and Classification of Breast Tumors in Ultrasonic Images Using Texture and Morphological Features," The Open Medical Informatics Journal, vol. 5, 26-37, 2011.

(13) Radscaper [http://radscaper.divinet.com]

(14) EZDicom [http://www.sph.sc.edu/comd/rorden/ezdicom.html]

(15) Rosse C and Mejino JL Jr., A reference ontology for biomedical informatics: the Foundational Model of Anatomy, J. Biomed Inform. 36:478-500, 2003.

(16) Rubin DL, Creating and curating a terminology for radiology: ontology modeling and analysis. J. Digit Imaging. 21:355-362, 2008.

(17) Y. Yu and S. T. Acton, "Speckle Reducing Anisotropic Diffusion,"IEEE Transactions on Image Processing, vol. 11, 1260-1270, 2002.

(18) S. T. Acton, "Locally monotonic diffusion," IEEE Trans. Signal Processing, vol. 48, pp. 1379-1389, May 2000.

(19) J. A. Noble and D. Boukerroui, "Ultrasound Image Segmentation: A Survey," IEEE transactions on medical imaging, vol. 25, 987-1010, 2006.

(20) A. M. F. Santos, R. M. d. Santos, P. M. A. C. Castro, E. Azevedo, L. Sousa, and J. M. R. S. Tavares, "A novel automatic algorithm for the segmentation of the lumen of the carotid artery in ultrasound B-mode images," Expert Systems with Applications, vol. 40, 6570-6579, 2013.

(21)D. Lin and J. Yu, "Otsu method and K-means," in 9th International Conference on Hybrid Intelligent Systems, 2009, 334-349.

(22) S. Saiyod and P. Wayalun, "A New Technique for Edge Detection of Chromosome G-BAND Images for Segmentation," in The 6th Asian Conference on Intelligent Information and Database Systems Bangkok, Thailand, 2014, 315-324.

(23) T. Chaoqiang and Y. Lizhen, "Research on collision detection algorithm Based on AABB-OBB Bounding Volume," in First International Workshop on Education Technology and Computer Science, 2009, 331-333. 